\title{
Relationship between ocular involvement and clinical manifestations, laboratory findings, and coronary artery dilatation in Kawasaki disease
}

\author{
Reza Shiari ${ }^{1} \cdot$ Mohsen Jari ${ }^{1} \cdot$ Saeed Karimi ${ }^{2,3} \cdot$ Omid Salehpour $^{4} \cdot$ Khosro Rahmani $^{1} \cdot$ Mehrnoosh Hassas Yeganeh $^{1}$. \\ Vadood Javadi Parvaneh ${ }^{1} \cdot$ Shabnam Hajian $^{1} \cdot$ Leyla Ghasemi $^{1} \cdot$ Sare Safi ${ }^{5}$
}

Received: 15 August 2019 / Revised: 27 October 2019 / Accepted: 23 November 2019 / Published online: 3 January 2020

(c) The Author(s), under exclusive licence to The Royal College of Ophthalmologists 2020

\begin{abstract}
Objectives To assess the incidence of ocular manifestations of Kawasaki disease (KD) in children and to evaluate the relationship between ocular manifestations and the other clinical manifestations, laboratory findings, and echocardiographic findings.

Methods Complete ophthalmologic examination and echocardiography were performed in 36 patients with KD during the acute phase before starting the treatment. Clinical manifestations and laboratory data including white blood cell (WBC) count, neutrophil-to-lymphocyte ratio, platelet count, erythrocyte sedimentation rate (ESR), C-reactive protein (CRP), and levels of alanine aminotransferase (ALT) and aspartate aminotransferase (AST) were obtained from all the patients. All the clinical and laboratory findings were compared between the group with ocular involvement and the one without ocular involvement.

Results The incidence of bilateral non-exudative conjunctivitis was $63.9 \%$. It was significantly higher in patients with skin rashes $(P<0.05)$. The incidence of uveitis was $36.1 \%$, which consisted primarily of grade $1+$ or $2+$ acute anterior uveitis. Neutrophil count and CRP levels were significantly higher in the uveitis group than in the group without uveitis $(P<0.05)$. Coronary artery dilatation showed significant correlation with uveitis $(P<0.05)$. Uveitis did not show a significant correlation with other clinical manifestations, ESR, ALT level, AST level, and platelet count $(P>0.05)$.

Conclusion In children with Kawasaki disease, uveitis is associated with coronary artery dilatation, higher neutrophil count, and higher CRP level.
\end{abstract}

Saeed Karimi

dr.saeedkarimi@gmail.com

1 Department of Paediatric Rheumatology, Mofid Children's Hospital, Shahid Beheshti University of Medical Sciences, Tehran, Iran

2 Ophthalmic Research Center, Shahid Beheshti University of Medicine Sciences, Tehran, Iran

3 Department of Ophthalmology, Torfeh Medical Center, Shahid Beheshti University of Medical Sciences, Tehran, Iran

4 Negah Specialty Ophthalmic Research Center, Shahid Beheshti University of Medical Sciences, Tehran, Iran

5 Ophthalmic Epidemiology Research Center, Shahid Beheshti University of Medicine Sciences, Tehran, Iran

\section{Introduction}

Kawasaki disease (KD) is the second most prevalent childhood vasculitis and the most prevalent acquired cause of heart diseases in children in many countries $[1,2]$. KD can be diagnosed by fever lasting for at least 5 days (which cannot be explained by any other disease) together with at least four additional criteria out of the five clinical manifestations that include bilateral non-exudative bulbar conjunctivitis, changes in oropharyngeal mucosa or strawberry tongue, unilateral anterior cervical lymphadenopathy, changes in the peripheral extremities, and polymorphous skin rash [3-5]. According to the guidelines of American Heart Association (AHA), incomplete KD is described as fever for at least 5 days together with 2-3 criteria out of the listed clinical manifestations $[4,5]$. KD can be accompanied by various ophthalmic manifestations including bilateral conjunctivitis, uveitis, iridocyclitis, superficial punctate 
Table 1 Distribution of ocular involvement in various parts of the affected eye in Kawasaki disease patients (total number of patients $=36$ ).

\begin{tabular}{lll}
\hline Characteristic & $\begin{array}{l}\text { Unilateral } \\
\text { involvement } \\
n(\%)\end{array}$ & $\begin{array}{l}\text { Bilateral } \\
\text { involvement } \\
n(\%)\end{array}$ \\
\hline $\begin{array}{l}\text { Conjunctivitis } \\
\begin{array}{l}\text { Scleral or conjunctival } \\
\text { scarring }\end{array}\end{array}$ & 0 & $23(63.9 \%)$ \\
$\begin{array}{l}\text { Acute anterior uveitis } \\
\begin{array}{l}\text { Acute intermediate } \\
\text { uveitis }\end{array}\end{array}$ & $2(5.5 \%)$ & 0 \\
\hline
\end{tabular}

keratitis, vitreous opacities, and papilledema [6]. Previous studies have shown that bilateral non-exudative bulbar conjunctivitis and acute uveitis are seen in about $80-90 \%$ and $20-80 \%$ of the KD patients, respectively. Although the ocular manifestations of KD improve spontaneously in most of the cases, scleral or conjunctival scarring, posterior synechiae, and blindness have been reported in rare cases [6-11].

The most important problem associated with $\mathrm{KD}$ is the involvement of coronary artery and the subsequent complications. In recent years, the number of patients who do not fulfil the diagnostic criteria of KD has increased [9]. Hence, it is difficult to decide the appropriate timing to start the treatment for $\mathrm{KD}$. On the other hand, delayed treatment is associated with complications related to coronary artery [4]. With increase in the number of cases of incomplete KD, uveitis has become a more important factor in the early diagnosis of incomplete KD. A few studies that reported the prevalence of ocular involvement in patients with Kawasaki disease reported the prevalence of conjunctivitis or acute uveitis without any grading of uveitis and its association with coronary artery involvement [10-17].

In the present study, we investigated the incidence of ocular involvement in $\mathrm{KD}$ and reported the grading of acute uveitis and its association with other clinical findings, laboratory data, and coronary artery dilatation in KD.

\section{Materials and methods}

Patients diagnosed with complete or incomplete KD from July 2018 to May 2019 were included in the study. All the patients were examined by an experienced ophthalmologist (Retina and Vitreous specialist) at the time of admission and 3 months after the admission. Ophthalmic examinations including slit lamp biomicroscopy and fundoscopy were performed in acute phase of the disease before starting the standard therapy. They were also performed 3 months later to evaluate the early ocular manifestations and the late sequelae of KD. Uveitis was diagnosed and graded using the Standardization of Uveitis Nomenclature grading scheme for anterior chamber cells and flare [18, 19].

Echocardiography was performed in all the patients by a paediatric cardiologist using an ultrasound device (Samsung HS70, Samsung Healthcare, Korea) at the time of diagnosis and 2 weeks after the diagnosis. For children aged less than 5 years, the coronary artery was considered dilated if the internal diameter of coronary artery lumen was more than 3 $\mathrm{mm}$ according to the AHA guidelines. For children aged 5 years or older, the coronary artery was considered dilated if the internal diameter of the lumen was more than $4 \mathrm{~mm}$ $[4,5]$. Demographic data including age and sex, clinical manifestations including bilateral non-exudative bulbar conjunctivitis, changes in oral mucosa, lips, or presence of strawberry tongue, unilateral anterior cervical lymphadenopathy, changes in the peripheral extremities, and polymorphous skin rash, laboratory values including erythrocyte sedimentation rate (ESR), C-reactive protein (CRP), white blood cell (WBC) count, neutrophil-to-lymphocyte ratio, platelet count, levels of alanine aminotransferase (ALT) and aspartate aminotransferase (AST), and echocardiographic findings were obtained and documented.

Statistical analyses were performed using IBM SPSS version 22 (IBM, New York, USA). The data were presented as mean \pm standard deviation or as percentages. Variables were compared between the two groups using the unpaired Student's $t$ test, Pearson chi-square test, Fisher's exact test, and one-way analysis of variance. $P$ values of $<0.05$ were considered statistically significant. The study was approved by the Institutional Research Ethics Committee, School of Medicine, Shahid Beheshti University of Medical Sciences and an informed consent was obtained from all subjects.

All patients were treated with intravenous immunoglobulin $(2 \mathrm{~g} / \mathrm{kg})$ and aspirin $(30 \mathrm{mg} / \mathrm{kg})$ as the first-line treatment, followed by aspirin $3-5 \mathrm{mg} / \mathrm{kg} / \mathrm{day}$ as a single daily dose. In patients resistant to intravenous immunoglobulin, a second dose of intravenous immunoglobulin $(2 \mathrm{~g} / \mathrm{kg})$ was administrated if fever was present for more than $36 \mathrm{~h}$ or the temperature was $\geq 38.0^{\circ} \mathrm{C}$.

All the patients with ocular involvement on initial examination were re-examined as needed, until the disease symptoms improved.

\section{Results}

During the study period, 36 patients were diagnosed with KD. Out of these, 25 cases $(69.4 \%)$ were diagnosed with complete KD and 11 cases $(30.6 \%)$ were diagnosed with incomplete KD. Twenty-one (58.3\%) patients were male and 15 patients $(41.7 \%)$ were female. 
Table 2 The SUN grading scheme for uveitis and ophthalmologic findings in the Kawasaki disease patients with uveitis.

\begin{tabular}{llcl}
\hline Grade & No. of cells & No. of patients $(\%)$ & Stage \\
\hline 0 & $<1$ & $0(0)$ & Acute \\
$0.5+$ & $1-5$ & $2(15.3 \%)$ & Acute \\
$1+$ & $6-15$ & $4(30.7 \%)$ & Acute \\
$2+$ & $16-25$ & $4(30.7 \%)$ & Acute \\
$3+$ & $26-50$ & $2(15.3 \%)$ & Acute \\
$4+$ & $>50$ & $1(7.5 \%)$ & Acute \\
& & Total $13(100 \%)$ & \\
\hline
\end{tabular}

SUN Standardization of Uveitis Nomenclature

The mean age of the patients was $30.41 \pm 4.35$ months (range: 3-108 months). During the acute phase of KD, 23 patients $(63.9 \%)$ showed bilateral non-exudative bulbar conjunctivitis. On ophthalmic examination, 13 patients $(36.1 \%)$ had acute uveitis. Acute anterior uveitis was seen in $86.4 \%$ of the uveitis cases, while $15.4 \%$ of the cases had intermediate uveitis. There was no case of posterior or panuveitis. No other ocular involvement was observed among the patients included in the study. At the 3-month follow-up visit, all the cases of uveitis and conjunctivitis had improved without any long-term ocular sequelae. Table 1 shows the distribution of ocular involvement in different parts of the affected eyes.

Most of the uveitis patients in the study had acute uveitis grade $1+$ or $2+$ according to the Standardization of Uveitis Nomenclature grading scheme for anterior chamber cells and flare (Table 2).

The incidence of ocular involvement including acute conjunctivitis and uveitis was not significantly different between the two genders $(P>0.05)$. There was no significant correlation between patients' age and ocular involvement $(P>0.05)$.

Twenty-four patients $(66.6 \%)$ had oral manifestations of Kawasaki disease. There was no significant correlation between ocular involvement and the oral manifestations of Kawasaki disease $(P>0.05)$.

Twenty-one patients (58.3\%) suffered from skin rashes. Acute conjunctivitis showed a significant correlation with skin rashes $(P<0.05)$ but there was no significant correlation between uveitis and skin rashes $(P>0.05)$.

Sixteen patients $(44.4 \%)$ had extremity changes and 11 patients $(30.5 \%)$ had cervical lymphadenopathy. There was no significant correlation between ocular involvement and extremity changes or cervical lymphadenopathy in $\mathrm{KD}$ patients $(P>0.05)$. Table 3 shows the relation of ocular involvement in $\mathrm{KD}$ with other clinical manifestations of the disease.

The incidence of conjunctivitis had no significant correlation with any of the laboratory values $(P>0.05)$ but the incidence of uveitis showed a significant correlation with CRP levels and neutrophil-to-lymphocyte ratio $(P<0.05)$.

Table 4 shows the relationship between coronary artery dilatation in $\mathrm{KD}$ patients and the incidence of ocular involvement. There was no significant correlation between conjunctivitis and coronary artery dilatation $(P>0.05)$. However, a direct relationship was observed between acute uveitis and coronary artery dilatation $(P<0.05)$.

\section{Discussion}

The present study showed that approximately two-thirds of the KD patients presented with bilateral non-exudative bulbar conjunctivitis in the acute phase (first 2 weeks), while acute uveitis was seen in approximately one-third of the patients. No other ocular manifestations were seen in this study. In terms of anatomical involvement of $\mathrm{KD}$ uveitis, the anterior segment was the most frequently affected part of the eyes (acute anterior non-granulomatous uveitis). Most of the patients showed grade $1+$ or $2+$ uveitis. In all the patients, the acute uveitis improved within 1 month after initial presentation and there were no longterm ocular complications observed at the 3-month followup. Previous studies have shown that the prevalence of bilateral non-exudative bulbar conjunctivitis in KD patients was about $60-90 \%$ in the acute phase of the disease [1-3, 13-17], which was similar to the results of the present study.

Choi et al. reported 110 cases of KD and found that $29 \%$ of the patients under study had acute uveitis during the acute phase of KD. Most of the uveitis cases in their study were graded as $1+$ and $2+$. Moreover, they showed that age, CRP, ESR, and neutrophil count in patients with uveitis were higher than in the patients without uveitis. Response to the treatment with intravenous immunoglobulin and coronary artery dilatation were not significantly different between the uveitis patients and the non-uveitis patients [16].

In the present study, bilateral non-exudative conjunctivitis and acute uveitis showed no significant correlation with patients' gender and age. However, Choi et al. suggested that the age of the uveitis patients was significantly higher than the age of the non-uveitis patients [16]. Genetic and environmental factors and sample size may be the reason for the difference between the two studies.

In the present study, ocular involvement showed no correlation with oral changes, changes in the extremities, and cervical lymphadenopathy, which are usual manifestations in the acute phase of KD. Acute bilateral conjunctivitis showed direct correlation with skin rashes in $\mathrm{KD}$, while there was no correlation between uveitis and skin rashes. 
Table 3 Relationship between ocular involvement and other clinical manifestations in Kawasaki disease patients.

\begin{tabular}{|c|c|c|c|c|c|c|}
\hline Characteristic & $\begin{array}{l}\text { Conjunctivitis }(+) \text { group } \\
(n=23)\end{array}$ & $\begin{array}{l}\text { Conjunctivitis (-) group } \\
(n=13)\end{array}$ & $P$ value* & $\begin{array}{l}\text { Uveitis }(+) \text { group } \\
(n=13)\end{array}$ & $\begin{array}{l}\text { Uveitis (-) group } \\
(n=23)\end{array}$ & $P$ value* \\
\hline Sex (male:female) & $14: 9$ & $7: 6$ & 0.68 & $8: 5$ & $13: 10$ & 0.76 \\
\hline Age (months) & $28.70 \pm 15.78$ & $39.08 \pm 23.17$ & 0.21 & $32.08 \pm 29.51$ & $32.70 \pm 20.33$ & 0.94 \\
\hline Oral changes & $17(73.9 \%)$ & $7(53.8 \%)$ & 0.28 & $11(84.6 \%)$ & $13(56.5 \%)$ & 0.14 \\
\hline Skin rash & $19(82.6 \%)$ & $2(15.3 \%)$ & 0.001 & $7(53.8 \%)$ & $14(60.8 \%)$ & 0.68 \\
\hline Extremity changes & $13(56.5 \%)$ & $3(23 \%)$ & 0.052 & $5(38.4 \%)$ & $11(47.8 \%)$ & 0.57 \\
\hline Cervical lymphadenopathy & $9(39.1 \%)$ & $2(15.3 \%)$ & 0.137 & $4(30.7 \%)$ & $7(30.4 \%)$ & 0.98 \\
\hline
\end{tabular}

$* P<0.05$ indicates statistically significant difference

Table 4 Relationship between laboratory findings, coronary artery dilatation, and ocular involvement in Kawasaki disease patients.

\begin{tabular}{|c|c|c|c|c|c|c|}
\hline Characteristic & $\begin{array}{l}\text { Conjunctivitis }(+) \text { group } \\
(n=23)\end{array}$ & $\begin{array}{l}\text { Conjunctivitis ( }-) \text { group } \\
(n=13)\end{array}$ & $P$ value* & $\begin{array}{l}\text { Uveitis }(+) \text { group } \\
(n=13)\end{array}$ & $\begin{array}{l}\text { Uveitis (-) group } \\
(n=23)\end{array}$ & $P$ value* \\
\hline WBC $\left(\times 10^{9} / \mathrm{L}\right)$ & $13.76 \pm 4.33$ & $14.24 \pm 11.19$ & 0.86 & $16.12 \pm 8.05$ & $12.69 \pm 7.59$ & 0.21 \\
\hline Platelets $\left(\times 10^{9} / \mathrm{L}\right)$ & $474 \pm 180.16$ & $564.62 \pm 271.97$ & 0.24 & $496.15 \pm 227.36$ & $513.17 \pm 218.09$ & 0.82 \\
\hline Neutrophils (\%) & $60.66 \pm 18.32$ & $51.63 \pm 16.77$ & 0.15 & $77.03 \pm 6.24$ & $46.30 \pm 11.96$ & 0.001 \\
\hline AST (IU/l) & $36.95 \pm 22.81$ & $39.23 \pm 16.27$ & 0.75 & $42.23 \pm 28.21$ & $35.26 \pm 14.65$ & 0.33 \\
\hline ALT (IU/l) & $44.60 \pm 37.89$ & $26.76 \pm 18.75$ & 0.12 & $51.15 \pm 46.11$ & $30.82 \pm 20.78$ & 0.07 \\
\hline $\mathrm{ESR}(\mathrm{mm} / \mathrm{hr})$ & $64.78 \pm 26.02$ & $55.23 \pm 41.40$ & 0.31 & $57.23 \pm 19.52$ & $63.65 \pm 30.92$ & 0.505 \\
\hline $\mathrm{CRP}(\mathrm{mg} / \mathrm{l})$ & $44.17 \pm 29.28$ & $24.76 \pm 30.10$ & 0.14 & $77.84 \pm 31.05$ & $14.17 \pm 17.15$ & 0.001 \\
\hline Coronary artery dilatation & $7(30.4 \%)$ & $3(23 \%)$ & 0.06 & $8(61.5 \%)$ & $2(8.6 \%)$ & 0.001 \\
\hline
\end{tabular}

$W B C$ white blood cell, $A S T$ aspartate aminotransferase, $A L T$ alanine aminotransferase, ESR erythrocyte sedimentation rate, $C R P C$-reactive protein $* P<0.05$ indicates statistically significant differences

This finding may be due to presumed similar inflammatory pathways in the ocular surface (conjunctiva) and the body surface (skin) in KD. There was no relationship between conjunctivitis and the laboratory findings including ESR, CRP, WBC, neutrophil-to-lymphocyte ratio, platelet count, ALT, and AST in KD, while uveitis was directly associated with CRP levels and neutrophil-to-lymphocyte ratio. Similarly, Choi et al. found that neutrophil-to-lymphocyte ratio in KD uveitis patients was significantly higher than in patients without uveitis. Moreover, CRP and ESR levels in the group with uveitis were higher than in KD patients without uveitis, though the findings were not statistically significant [16].

Kawasaki disease is the most common acquired heart disease in children. Delayed treatment can cause cardiac complications including coronary artery dilatation and aneurysm, myocardial infarction, pericardial effusion, and valvular regurgitation [4]. The results of the present study showed that coronary artery dilatation in acute phase of KD showed no significant correlation with acute bilateral conjunctivitis, while there was statistically significant relationship between acute uveitis and coronary artery dilatation. Some authors have presented arguments against this direct relationship. Lee et al. showed that in incomplete $\mathrm{KD}$, the risk of coronary artery lesions was lower in uveitis patients, as the presence of uveitis was helpful in the early diagnosis and treatment of incomplete KD [17]. In the present study, the treatment was not delayed in the uveitis patients. Hence, the presence of uveitis may be associated with higher severity of the disease and subsequently, with a higher risk of coronary artery dilatation. Contrary to the results of the present study, Choi et al. found that coronary artery dilatation was not significantly different between the uveitis and the non-uveitis groups. However, in that study, the mean age of the uveitis group was higher than the control group and although the clinical characteristics were similar, the uveitis group showed a higher incidence of complete Kawasaki disease [16].

It is our belief that acute uveitis may be considered as one of the essential diagnostic criteria for Kawasaki disease. Coronary aneurysm is the most dangerous and important complication of KD and the goal of early diagnosis and treatment of $\mathrm{KD}$ is to prevent coronary artery dilatation. Hence, the relationship between acute uveitis and coronary artery dilatation is highly important. It is difficult to decide the best timing for ophthalmic evaluation in KD. There is no specific guideline to determine the timing of slit lamp examination in KD. This issue can be investigated in future studies. The strong point of our study is that all the patients with $\mathrm{KD}$ underwent ophthalmologic 
examinations. However, the study has some limitations such as low sample size, single institution, and short duration of follow-up.

In conclusion, we found that acute uveitis showed a significant correlation with coronary artery dilatation in $\mathrm{KD}$. We recommend slit lamp examination for all patients with KD to determine the presence of uveitis. Since early diagnosis and treatment of KD can reduce cardiac complications, we recommend that the paediatricians should cooperate with the ophthalmologists while managing patients who are suspected of having KD.

\section{Summary}

\section{What was known before}

- A few studies that reported the prevalence of ocular involvement in patients with Kawasaki disease reported the prevalence of conjunctivitis or acute uveitis without any grading of uveitis and its association with coronary artery involvement.

\section{What this study adds}

- In children with Kawasaki disease, uveitis is associated with coronary artery dilatation, higher neutrophil count, and higher CRP level.

Acknowledgements We thank Ms. Maryam Yousefi for her valuable contribution to the study.

\section{Compliance with ethical standards}

Conflict of interest The authors declare that they have no conflict of interest.

Publisher's note Springer Nature remains neutral with regard to jurisdictional claims in published maps and institutional affiliations.

\section{References}

1. Nakamura Y, Yashiro M, Uehara R, Oki I, Kayaba K, Yanagawa $\mathrm{H}$. Increasing incidence of Kawasaki disease in Japan: nationwide survey. Pediatr Int. 2008;50:287-90.
2. Gerding R. Kawasaki disease: a review. J Pediatr Health Care. 2011;25:379-87.

3. Alexoudi I, Kanakis M, Kapsimali V, Vaiopoulos G. Kawasaki disease: current aspects on aetiopathogenesis and therapeutic management. Autoimmun Rev. 2011;10:544-7.

4. Newburger JW, Takahashi M, Gerber MA, Gewitz MH, Tani LY, Burns JC, et al. Diagnosis, treatment, and long-term management of Kawasaki disease: a statement for health professionals from the Committee on Rheumatic Fever, Endocarditis, and Kawasaki Disease, Council on Cardiovascular Disease in the Young, American Heart Association. Circulation. 2004;110:2747-71.

5. McCrindle BW, Rowley AH, Newburger JW, Burns JC, Bolge AF, Gewitz M. Diagnosis, treatment, and long-term management of Kawasaki disease. A scientific statement for health professionals from the American Heart Association. Circulation. 2017;135: e927-e999. https://doi.org/10.1161/CIR.000000000000048

6. Ohno S, Miyajima T, Higuchi M, Yoshida A, Matsuda H, Saheki Y, et al. Ocular manifestations of Kawasaki's disease (mucocutaneous lymph node syndrome). Am J Ophthalmol. 1982; 93:713-7.

7. Burke MJ, Rennebohm RM. Eye involvement in Kawasaki disease. J Pediatr Ophthalmol Strabismus. 1981;18:7-11.

8. Burke MJ, Rennebohm RM, Crowe W, Levinson JE. Follow-up ophthalmologic examinations in children with Kawasaki's disease. Am J Ophthalmol. 1981;91:537-9.

9. Freeman AF, Shulman ST. Kawasaki disease: summary of the American Heart Association guidelines. Am Fam Physician. 2006;74:1141-8.

10. Madhusudan S, Singh S, Suri D, Gupta A, Gupta A. Acute anterior uveitis as the presenting feature of Kawasaki disease. Indian J Pediatr. 2014;81:415.

11. Cunningham ET Jr. Uveitis in children. Ocul Immunol Inflamm. 2000;8:251-61.

12. Rennebohm RM, Burke MJ, Crowe W, Levinson JE. Anterior uveitis in Kawasaki's disease. Am J Ophthalmol 1981;91:535-7.

13. Jacob JL, Polomeno RC, Chad Z, Lapointe N. Ocular manifestations of Kawasaki disease (mucocutaneous lymph node syndrome). Can J Ophthalmol 1982;17:199-202.

14. Offret H. Disciform keratitis and Kawasaki's disease. J Fr Ophtalmol 1993;16:114-6.

15. Erdem E, Kocabas E, Taylan HS, ÖzgürO, Yagmur M, Ersoz TR. Crystalline-like keratopathy after intravenousImmunoglobulin therapy with incomplete Kawasaki disease: case report and literature review. Case Rep. Ophthalmol Med. 2013;2013:621952. https://doi.org/10.1155/2013/621952.

16. Choi HS, Lee SB, Kwon JH, Kim HS, Sohn S, Hong YM. Uveitis as an important ocular sign to help early diagnosis in Kawasaki disease. Korean J Pdiatr. 2015;58:374-9.

17. Lee KJ, Kim HJ, Kim MJ, Yoon JH, Lee EJ, Lee JY, et al. Usefulness of anterior uveitis as an additional tool for diagnosing incomplete Kawasaki disease. Korean J Pediatr. 2016;59:174-7.

18. Guney E, Tugal-Tutkun I. Symptoms and signs of anterior uveitis. US Ophthalmic Rev. 2013;6:33-37.

19. Jabs DA, Nussenblatt RB, Rosenbaum JT. Standardization of Uveitis Nomenclature (SUN) Working Group. Standardization of uveitis nomenclature for reporting clinical data. Results of the First International Workshop. Am J Ophthalmol. 2005;140: 509-16. 